\title{
Vínculo de familiares de recém-nascidos internados em uma Unidade de Terapia Intensiva Neonatal
}

\author{
Bonding of family members of newborns admitted to a Neonatal Intensive Care Unit
}

\author{
Vinculación de familiares de recién nacidos ingresados em uma Unidad de Cuidados Intensivos Ne- \\ onatales
}
Lethícia de Oliveira de Souza ${ }^{1 *}$, Jannaína Sther Leite Godinho Silva², Adiel Queiroz Ricci , Alessandra da Silva Souza ${ }^{4}$, Eliara Adelino da Silva ${ }^{5}$, Geisa Sereno Velloso da Silva ${ }^{6}$.

Como citar esse artigo. de Souza, LO; Silva, JSLG; Ricci, AQ; Souza, AS; da Silva, EA; da Silva, GSV. Vínculo de familiares de recém-nascidos internados em uma Unidade de Terapia Intensiva Neonatal. Revista Pró-UniverSUS. 2021 Jul./Dez.; 12 (2)SUPLEMENTO: 27-31.

\section{Resumo}

A Unidade de Terapia Intensiva Neonatal é um espaço em que o princípio entre a vida e a morte é sucinto. As informações do melhoramento do bebê são dadas diariamente, em que se evita fazer estimativas para além daquele dia. Para que a mãe possa dar apoio psíquico ao seu bebê, a equipe da Unidade de Terapia Intensiva Neonatal precisa coibir seus próprios medos e lutos, uma vez que vive com o bebê internado em uma vigorosa relação de cuidado, dessa maneira, o zelo com a mãe será mantido. A pesquisa foi realizada usando as bases de dados "Scientific Eletonic Library Online (SCIELO)", "LILACS", "BDENF" e "MEDLINE" no período de setembro a dezembro de 2020. O objetivo desse estudo é identificar o vínculo dos familiares de recém-nascidos submetidas à internação nas Unidades de Terapia Intensiva Neonatal. Trata-se de uma pesquisa qualitativa, exploratória, descritiva, realizada através de uma revisão integrativa de literatura. Foi observado durante a análise dos artigos para estudo, que os familiares são as pessoas que mais se submetem a sentimentos de impotência, por não conseguirem fazer nada para ajudar o filho quando o mesmo se encontra em uma situação de risco, e tristeza, por não poderem levar seu filho para a casa após o seu nascimento. Destacou-se durante esse estudo, a importância em incluir a equipe de saúde da UTIN para prestar os cuidados necessários aos recém-nascidos baseando-se nos protocolos e rotinas assistencias, e também, prestando o devido zelo aos familiares que tanto sofrem de aflição e ansiedade, ao ver seu filho internado.

Palavras-chave: Enfermagem; UTI Neonatal; Profissão; Vínculo de familiares.

\begin{abstract}
The Neonatal Intensive Care Unit is a space in which the principle between life and death is succinct. Information about the baby's improvement is given daily, avoiding making estimates beyond that day. In order for the mother to provide psychic support to her baby, the team at the Neonatal Intensive Care Unit needs to restrain her own fears and grief, since she lives with the baby hospitalized in a vigorous care relationship, in this way, the zeal with the child. mother will be kept. The research was carried out using the databases "Scientific Eletonic Library Online (SCIELO)", "LILACS", "BDENF" and "MEDLINE" from September to December 2020. The objective of this study is to identify the link of family members of newborns submitted to hospitalization in Neonatal Intensive Care Units. It is a qualitative, exploratory, descriptive research, carried out through an integrative literature review. It was observed during the analysis of the articles for study, that family members are the people who most submit to feelings of helplessness, for not being able to do anything to help the child when he is in a risky situation, and sadness, for not to be able to take their child home after their birth. During this study, the importance of including the NICU health team to provide the necessary care to newborns based on the protocols and care routines was highlighted, as well as paying due care to family members who suffer from affliction and anxiety when seeing your child hospitalized.
\end{abstract}

Keywords: Nursing; Neonatal ICU; Profession; Family Link.

${ }^{1 *}$ Acadêmica do Curso de Enfermagem, Universidade de Vassouras. RJ, Brasil. E-mail: lethicia epf@hotmail.com ORCID: https://orcid.org/0000-0002-1634-5754. ${ }^{2}$ Mestre. Docente do Curso de Enfermagem da Universidade de Vassouras. RJ, Brasil. E-mail: jjasther@gmail.com ORCID: https://orcid.org/0000-0002-8308-2093. ${ }^{3}$ Doutorando em Estudos de Linguagem pelo Programa de Estudos de Linguagem da UFF. Mestre em Estudos de Linguagem pelo Programa de Estudos de Linguagem da UFF. Docente do Curso de Enfermagem da Universidade de Vassouras, RJ, Brasil. E-mail: adielricci@gmail.com ORCID: https://orcid.org/0000-0003-2761-2499. ${ }^{4}$ Mestre. Especialista em Neonatalogia. Docente do Curso de Enfermagem da Universidade de Vassouras. RJ, Brasil. E-mail: alesouza22@yahoo.com.br ORCID: https://ORCID.org/0000-0002.9009-9774 . ${ }^{5}$ Mestre. Terapeuta Holística. Mestre em Ciências da Saúde e Meio Ambiente. Docente do Curso de Enfermagem da Universidade de Vassouras. RJ, Brasil. E-mail: eliaraadelinos@uol.com.br ORCID: https:// orcid.org/0000-0003-4387-6662. ${ }^{6}$ Mestre em Enfermagem pela Universidade Federal do Rio de Janeiro. Docente do Curso de Enfermagem da Universidade de Vassouras. RJ, Brasil. E-mail: geisa.velloso@hotmail.com ORCID: https://orcid.org/0000-0003-0304-8010.

* Email de correspondencia: lethicia_epf@hotmail.com 


\section{Resumen}

La Unidad de Cuidados Intensivos Neonatales es un espacio en el que el principio entre la vida y la muerte es sucinto. La información sobre la mejoría del bebé se da diariamente, evitando hacer estimaciones más allá de ese día. Para que la madre brinde apoyo psíquico a su bebé, el equipo de la Unidad de Cuidados Intensivos Neonatales necesita contener sus propios miedos y dolor, ya que vive con el bebé hospitalizado en una relación de cuidado vigorosa, de esta manera, el celo con la madre del niño se mantendrá. La investigación se realizó utilizando las bases de datos "Scientific Eletonic Library Online (SCIELO)", "LILACS", "BDENF” y “MEDLINE” de septiembre a diciembre de 2020. El objetivo de este estudio es identificar el vínculo de los familiares de recién nacidos sometidos a hospitalización en Unidades de Cuidados Intensivos Neonatales. Se trata de una investigación cualitativa, exploratoria, descriptiva, realizada a través de una revisión integradora de la literatura. Se observó durante el análisis de los artículos a estudio, que los familiares son las personas que más se someten a sentimientos de impotencia, por no poder hacer nada para ayudar al niño cuando se encuentra en una situación de riesgo, y tristeza, por no hacerlo. para poder llevar a su hijo a casa después de su nacimiento. Durante este estudio se destacó la importancia de incluir al equipo de salud de la UCIN para brindar la atención necesaria a los recién nacidos en base a los protocolos y rutinas de atención, así como prestar la debida atención a los familiares que padecen aflicción y ansiedad al ver a su hijo hospitalizado.

Palabras clave: Enfermería; UCI Neonatal; Profesión; Vínculo Familiar

\section{Introdução}

A Unidade de Terapia Intensiva Neonatal (UTIN) determina-se pelo acolhimento de recém-nascidos $(\mathrm{RN})$, desde o seu nascimento até o primeiro mês de vida, que se mantém internados por tempo crucial para o avanço de sua saúde. A UTIN coadjuva para o tratamento das moléstias neonatais e diminuição da letalidade, associando a ciência ao saber específico dos profissionais. A internação de um neonato na UTIN é definida primitivamente pela ruptura do habitual nascer de um bebê saudável, o qual se mantém de forma positiva desde os primeiros instantes de vida ${ }^{1}$.

A Unidade de Terapia Intensiva Neonatal é um espaço em que o princípio entre a vida e a morte é sucinto. As informações do melhoramento do bebê são dadas diariamente, em que se evita fazer estimativas para além daquele dia. Para que a mãe possa dar apoio psíquico ao seu bebê, a equipe da Unidade de Terapia Intensiva Neonatal precisa coibir seus próprios medos e lutos, uma vez que vive com o bebê internado em uma vigorosa relação de cuidado, dessa maneira, o zelo com a mãe será mantido².

$\mathrm{O}$ nascimento de um recém-nascido prematuro ou a constatação de enfermidades é marcado pelo afastamento físico entre este e sua mãe, evoluindo para situações difíceis e sentimentos negativos. Unido a esses sentimentos está à dificuldade de adaptação ao cotidiano estressante da UTIN e as dificuldades que intervêm à busca da supervivência do filho. Neste meio delicado e caótico, adentram sentimentos de medo, tristeza, culpa e insegurança. Mais além das questões relativas para tais perspectivas, o nascimento de um filho representa também o começo de uma série de mudanças na família, seja em razão da obtenção de novas funções e responsabilidades pelos componentes da família, seja pelo afogo e ansiedade de que algo possa abalar o curso planejado, principalmente quando acontece um nascimento de um neonato prematuro e/ou de risco. Em relação à análise médica, se diferenciam os problemas clínicos, situação de emergência após o parto, prematuridade forte e necessidade de cirurgia ${ }^{3}$.

No momento da assistência ao prematuro nas UTIN, algumas práticas têm passado por transformações. Algumas intervenções têm sido efetivadas nas unidades neonatais para operacionalizar os serviços da equipe de saúde, sendo eles: a inserção de grupos de apoio e permissão de visitas dos familiares, a aproximação dos pais ao filho internado, e o incentivo à mãe na participação do cuidado do bebê no momento de tomar decisões importantes ${ }^{4}$.

A esperança de levar o recém-nascido para casa é tida como um perfeito carinho pela família desde o começo da gestação e amparado praticamente dia a dia pelos pais até o nascimento. Desse modo, a partir do momento que os pais recebem a notícia de que seu filho precisará de assistência especializada, em uma UTIN, os pais são abalados por outros sentimentos distantes daqueles concebidos pelo nascimento de um bebê de risco, sendo elas a agonia, a ansiedade e a intranquilidade quanto ao futuro que, de repente, se torna indefinido e ameaçado pelo pânico e pela culpabilidade de deixar seu filho internado em uma Unidade de Terapia Intensiva Neonatal e não o levar para casa.

Com base no que foi apresentado a questão que norteou o estudo foi: Como se estabelece o vínculo de familiares de recém-nascido internados em Unidade de Terapia Intensiva Neonatal.

Diante do exposto o objetivo do estudo foi descrever o vínculo de familiares de recém-nascidos internados em uma Unidade de Terapia Intensiva Neonatal a partir da literatura científica ${ }^{5}$.

\section{Metodologia}

Trata-se de um estudo qualitativo, exploratório descritivo que foi realizado através de uma revisão 
integrativa de literatura.

A revisão integrativa, combina diversas metodologias para auxiliar a ausência de exatidão, tendo que ser acompanhada dentro dos padrões de certezas metodológicas ${ }^{6}$.

A pesquisa descritiva exibe atributos de uma estipulada população ou fenômeno, determina ligações entre variáveis e define sua natureza. A pesquisa não tem o comprometimento de explicar os fenômenos que retrata, apesar de servir de embasamento para tal explicação ${ }^{7}$.

A pesquisa qualitativa abrange uma perspectiva esclarecedora do mundo, o que quer dizer que seus exploradores estudam as coisas em seu contexto originário, tentando compreender os fatos em termos dos conceitos que as pessoas a eles confirmam ${ }^{8}$.

A pesquisa foi realizada usando as bases de dados "Scientific Eletonic Library Online (SCIELO)", "LILACS", "BDENF" e "MEDLINE" no período de setembro a dezembro de 2020.

As buscas ocorreram por meio das junções dos descritores "Enfermagem AND Atenção Básica AND Políticas Públicas" "Enfermagem and Primary Care and Public policy" obteve-se um resultado de 45 artigos, dentre os quais foram selecionados apenas 1 artigo que estava relacionado com o tema após a leitura do título e resumo. Os demais foram descartados pelos motivos: 1 por estar repetido e 43 por não atenderem o objetivo da pesquisa. Devido ao número insuficiente de artigos, se fez necessário uma nova pesquisa. Para um novo levantamento de dados foram relacionados os seguintes descritores: "Enfermagem AND Atenção Básica de Saúde" "Nursing AND Primary Health Care" no banco de dados citado anteriormente no qual foram encontrados 120 artigos, dentre os quais foram utilizados 08 artigos. Os demais foram descartados pelos motivos: 1 por ter sido publicado a mais de 10 anos, 10 por não estarem disponíveis on-line na íntegra no banco de dados, 10 por estarem repetidos e 81 por não atenderem aos objetivos da pesquisa. Para um novo levantamento de dados, foram relacionados os seguintes escritores: "Enfermagem" AND "Unidade de Terapia Intensiva Neonatal" onde foram encontrados 115 artigos, dentre os quais foram utilizados 02 artigos para o presente estudo. Os demais foram descartados pelo motivo de não atenderem o objetivo do estudo.

Foram selecionados para esta pesquisa 10 artigos compondo o conteúdo do presente estudo.

\section{Resultados e Discussão}

No quadro 1, pode-se observar os periódicos dos artigos analisados para o presente estudo.

Observando-se a área de conhecimento dos periódicos, verificou-se que as publicações analisadas na maior parte dos estudos que abordavam o vínculo de familiares de recém-nascidos internados em uma Unidade de Terapia Intensiva Neonatal foram publicadas na Revista Brasileira de Enfermagem totalizando um total de três artigos conforme a Quadro 1. A Revista Brasileira de Enfermagem é um espaço científico destinado a publicações da área de Enfermagem o que justifica um número maior de publicação sobre o objetivo do trabalho.

Quadro 1. Identificação dos artigos conforme seus periódicos e número total de artigos analisados.

\begin{tabular}{|l|c|}
\hline \multicolumn{1}{|c|}{ Periódicos } & Número de Artigos \\
\hline Revista de Economia e Sociologia Rural & 01 \\
\hline Revista Pró-Univer SUS & 01 \\
\hline Revista Gaúcha de Enfermagem & 01 \\
\hline $\begin{array}{l}\text { Revista de Pesquisa: O Cuidado é } \\
\text { Fundamental On-Line }\end{array}$ & 01 \\
\hline Revista Brasileira de Enfermagem & 03 \\
\hline Revista de Enfermagem UFPE on-line & 02 \\
\hline $\begin{array}{l}\text { Revista do Programa de Pós-graduação } \\
\text { em Controladoria e Contabilidade } \\
\text { da UFRGS }\end{array}$ & 01 \\
\hline Total & $\mathbf{1 0}$ \\
\hline
\end{tabular}

Observou-se durante a análise dos artigos para estudo, que os familiares são as pessoas que mais se submetem a sentimentos de impotência, por não conseguirem fazer nada para ajudar o filho quando o mesmo se encontra em uma situação de risco, e tristeza, por não poderem levar seu filho para a casa após o seu nascimento.

O processo de internção do neonato, se divide em fases, onde o familiar vai se descobrindo no decorrer da evolução da mesma. A princípio, tem a fase de rejeição que se inicia no momento do atendimento e se insiste ao decorrer dos dias que a procedem, num período indefinido 9 .

Ela acontece pois ao decorrer da gestação, o vínculo entre mãe e filho se fortifica. A mãe se organiza para a recepção do seu bebê saudável e idealiza como será o primeiro encontro com o mesmo 9 .

É importante ressaltar o dever do reconhecimento da equipe de Enfermagem, da promoção de um cuidado humanizado. É preciso prestar acolhimento aos familiares, na UTIN, a fim de que seja estabelecido conforto, segurança e revigoração do vínculo entre os pais e o bebê $\hat{e}^{10}$.

A partir do momento em que seu o recém-nascido fica internado em uma UTIN, a mãe se desconecta da sua rotina, se distancia do seu trabalho, das suas funções como mãe em relação a outros filhos e também da sua vida de esposa, quando se tem um companheiro. A vida se transforma e ela passa a viver um novo cotidiano, tendo que se adaptar a essa fase ${ }^{11}$.

O componente fundamental e único no cuidado do recém-nascido na UTIN é a família, sobretudo pela sua representação para o conforto, evolução e 
saúde do neonato. O princípio da humanização pela sua representação em saúde foi contemplado como um enfoque para assumir riscos, como o momento de entubação do RN no colo da sua mãe ${ }^{12}$.

Deste modo, a relação e o diálogo entre o profissional de saúde e os familiares são significativos para entender o que está se sucedendo com o seu filho. Ainda que existam muitas sensações de rejeição e confusão, os pais não se identificam de uma maneira que estejam sendo excluídos quando não se obtém esclarecimento e encorajamento à ter proximidade física com o seu bebê e entender que ele está sendo assistido por pessoas qualificadas é um ponto valoroso para eles ${ }^{11}$.

Fase de Consolidação: Ao longo da fase de consolidação, a adequação dos pais à existência do seu bebê na UTIN e ao seu atual dever maternal e paternal é fundamental. Os pais passam de serem pessoas observadoras para serem pessoas atuantes. Eles carecem de distintas maneiras de conhecimento em opostas fases de sua internação infanti1 ${ }^{9}$.

O componente fundamental e único no cuidado do recém-nascido na UTIN é a família, sobretudo pela sua representação para o conforto, evolução e saúde do neonato. $\mathrm{O}$ princípio da humanização pela sua representação em saúde foi contemplado como um enfoque para assumir riscos, como o momento de entubação do RN no colo da sua mãe ${ }^{12}$.

Fase da Alta: Independentemente da vontade dos pais de levarem o seu filho para casa, a etapa de organização para a alta da UTIN é definida pelos sentimentos de felicidade e temor. Ir embora logo após um contato com profissionais qualificados na UTIN pode ser uma adversidade?.

Os enfermeiros prestam total apoio aos pais e os preparam para a alta do seu bebê, ainda que haja muita comoção e dúvida, os pais provam esta etapa como uma vivência proveitosa ${ }^{13}$.

Destaca-se que a ligação definida que atua na UTIN é de imensa relevância para que os pais sintamse mais resguardados em relação ao quadro do seu filho, especialmente, ao grupo da Enfermagem, que se mantém todo o tempo próximo do netonato e induz a existência dos pais ao lado do seu bebê ${ }^{11}$.

Observa-se que a equipe de Enfermagem é a conexão da família com o neonato, já que, através da relação com os integrantes da equipe multiprofissional e da assistência prestada ao bebê, os pais se vêem incluídos no processo de internação do seu filho, reconhecendo o cuidado e a atenção dos profissionais, construindo assim, um vínculo de união ${ }^{10}$.

\section{Conclusão}

O estudo buscou identificar o sentimento dos familiares frente as internações de seus filhos na Unidade de Terapia Intensiva Neonatal. Dentre as buscas realizadas para o presente estudo, pode-se observar que o tema sobre vínculo de familiares de recém-nascidos internados em uma Unidade de Terapia Intensiva Neonatal não tem muito enfoque nos sentimentos dos familiares, e sim nas patologias e sofrimentos para o recém-nascido.

Durante a pesquisa, constatou-se que tanto o neonato quanto os familiares necessitam de assistência da equipe de Enfermagem sendo o recém-nascido com cuidados patológicos, e os familiares com assistência psicológica e afetiva para tornar a estadia do bebê um período de bem estar e acolhedor para ambos.

Apesar das dificuldades de encontrar artigos focados no presente assunto, considero alcançados os objetivos de identificar o vínculo de familiares de recém-nascidos internados em uma Unidade de Terapia Intensiva Neonatal, que condizem com os sentimentos de tristeza, impotência, insegurança e angústia dos pais que são submetidos a conviver com o processo de internação do seu bebê. Este estudo contribuiu para a área de Unidade de Terapia Intensiva Neonatal, na Enfermagem.

\section{Referências}

1. Brasil. Ministério da Saúde. Portaria GM/MS No 3432 , de 12 de agosto de 1998, que estabelece critérios de classificação para as Unidades de Tratamento Intensivo - UTI. Diário Oficial da União. Brasília, n. 3432, 13 de agosto de 1998. Recuperado em 21 out. 2008. Disponível em: http://bvsms. saude.gov.br/bvs/saudelegis/gm/1998/prt3432_12_08_1998.html

2. Exequiel N, Pereira MVM, Gabatz RIB, Vaz JC, Hirschmann B, Hirschmann R. Vivências da família do neonato internado em Unidade de Terapia Intensiva. Revista Enfermagem Atual. 2019 Ago; 88-27.

3. Almeida CR, Morais AC, Lima KDFLS, Silva ACOC. Cotidiano de mães acompanhantes na Unidade de Terapia Intensiva Neonatal. Revista Enfermagem UFPE on-line. 2018 Jul; 12(7):1949-56.

4. Veronez M, Borghesan NAB, Corrêa DAM, Hiharashi IH. Experiência de mãe de bebês prematuros do nascimento à alta: Notas de diários de campo. Rev. Gaúcha Enferm. 2017 Ju; 38(2): e60911.

5. Gaíva MAM, Scochi CGS. A participação da família no cuidado ao prematuro em UTI Neonatal. Rev. Brasileira de Enferm. 2005 ago; 58 (4): 444-448.

6. Gomes JMM, Fernandes L. Relatórios de pesquisa nas ciências sociais: características e modalidades de investigação. Porto Alegre; 2003 v. 3 n. 4.

7. Augusto CA, Souza JP de, Dellagnelo EHL, Cario SAF. Pesquisa qualitativa: rigor metodológico no tratamento da teoria dos custos de transação em artigos apresentados nos congressos da Sober. Rev. Econ. Sociol. Rura. 2013oct-dez. vol. 51 n. 4.

8. Frello AT, Carraro TE. Enfermagem e a relação com as mães de neonatos em Unidade de Terapia Intensiva Neonatal. 2012 jun; 65(3): 514521

9. Almeida CR, Morais AC, Lima KDFLS, Silva ACOC. Cotidiano de mães acompanhantes na Unidade de Terapia Intensiva Neonatal. Revista Enfermagem UFPE on-line. 2018 Jul; 12(7):1949-56.

10. Marski BSL, Facio BC, Ichisato SMT, Barba PCSD, Wernet M. Developmental Care: assistance of nurses from Neonatal Intensive Care Units. Rev Bras. Enferm. 2018;71(6):2758-66. 
11. Antunes BS, Cardoso CP, Padoin MMS, Trojahn TC, Rodrigues AP, Tronco CS. Internação do recém-nascido na Unidade Neonatal: significado para a mãe. Rev Rene. 2014 set-out; 15(5):796-803.

12. Gomes RNS; Filha FSSC; Portela NLC. Avaliação da influência do abandono da assistência pré-natal na mortalidade fetal e neonatal. Rev. Fund Care Online. 2017 abr/jun; 9(2):416-421

13. Lima, GR; Silva, JSLG. Vivência dos profissionais de enfermagem perante a morte neonatal. Revista Pró-UniverSUS. 2019 jan-jul.; 10 (1): 3841 